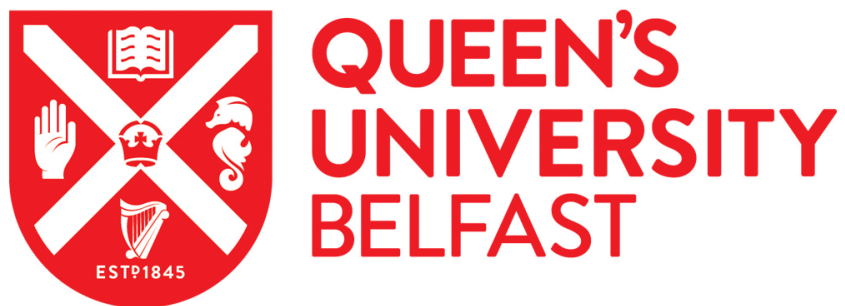

\section{A Fast Analysis Method for the Groove Gap Waveguide Using Transmission Line Theory}

Berenguer, A., Fusco, V., Ferrnado-Rocher, M., \& Boria, V. E. (2016). A Fast Analysis Method for the Groove Gap Waveguide Using Transmission Line Theory. In Proceedings of 10th European Conference on Antennas \& Propagation (EUCAP 2016) Institute of Electrical and Electronics Engineers Inc.. https://doi.org/10.1109/EuCAP.2016.7481433

Published in:

Proceedings of 10th European Conference on Antennas \& Propagation (EUCAP 2016)

Document Version:

Peer reviewed version

Queen's University Belfast - Research Portal:

Link to publication record in Queen's University Belfast Research Portal

\section{Publisher rights}

() 2016 by the European Association on Antennas and Propagation (EurAAP). All rights reserved.

Personal use of this material is permitted. Permission from IEEE must be obtained for all other uses, in any current or future media, including reprinting/republishing this material for advertising or promotional purposes, creating new collective works, for resale or redistribution to servers or lists, or reuse of any copyrighted component of this work in other works.

\section{General rights}

Copyright for the publications made accessible via the Queen's University Belfast Research Portal is retained by the author(s) and / or other copyright owners and it is a condition of accessing these publications that users recognise and abide by the legal requirements associated with these rights.

\section{Take down policy}

The Research Portal is Queen's institutional repository that provides access to Queen's research output. Every effort has been made to ensure that content in the Research Portal does not infringe any person's rights, or applicable UK laws. If you discover content in the Research Portal that you believe breaches copyright or violates any law, please contact openaccess@qub.ac.uk. 


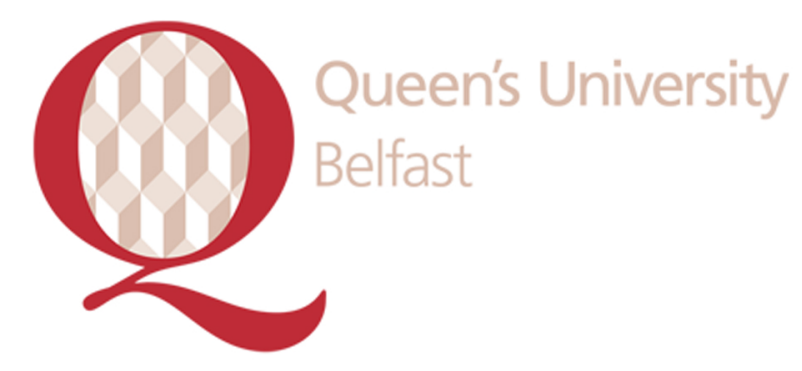

\section{A Fast Analysis Method for the Groove Gap Waveguide Using Transmission Line Theory}

Berenguer, A., Fusco, V., Ferrnado-Rocher, M., \& Boria, V. (2016). A Fast Analysis Method for the Groove Gap Waveguide Using Transmission Line Theory. 1588 - 1593. Paper presented at 10th European Conference on Antennas \& Propagation, Davos, Switzerland, Davos, Switzerland.

Document Version:

Early version, also known as pre-print

Queen's University Belfast - Research Portal:

Link to publication record in Queen's University Belfast Research Portal

\section{General rights}

Copyright for the publications made accessible via the Queen's University Belfast Research Portal is retained by the author(s) and / or other copyright owners and it is a condition of accessing these publications that users recognise and abide by the legal requirements associated with these rights.

\section{Take down policy}

The Research Portal is Queen's institutional repository that provides access to Queen's research output. Every effort has been made to ensure that content in the Research Portal does not infringe any person's rights, or applicable UK laws. If you discover content in the Research Portal that you believe breaches copyright or violates any law, please contact openaccess@qub.ac.uk. 


\title{
A Fast Analysis Method for the Groove Gap Waveguide Using Transmission Line Theory
}

\author{
Antonio Berenguer ${ }^{1}$, Vincent Fusco $^{2}$, Miguel Ferrando-Rocher ${ }^{1}$, Vicente E. Boria ${ }^{1}$, \\ ${ }^{1}$ Instituto de Telecomunicaciones y Aplicaciones Multimedia (iTEAM) \\ Universitat Politècnica de València, Valencia (46022) Spain, anbever@iteam.upv.es \\ ${ }^{2}$ The Institute of Electronics, Communications and Information Technology (ECIT) \\ Queen's University of Belfast, Belfast (BT3 9DT) United Kingdom
}

\begin{abstract}
The Groove Gap Waveguide (GGW) shows a behavior similar to the classical rectangular waveguide (RWG), but it is formed by two pieces which do not require metal contact. This feature suggests the GGW as a suitable alternative to the RGW for mm-wave frequencies, where ensuring the proper metal contact according to the wavelength size results challenging. Nevertheless, there is a lack of effective analysis tools for the complex GGW topology, and assuming a direct equivalence between the RGW and the GGW is too rough, so that dilatory full-wave simulations are required. This work presents a fast analysis method based on transmission line theory, which establishes the proper correspondence between the GGW and the RWG. In addition, below cutoff behavior of the GGW is studied for the first time. Several numerical tests and two manufactured prototypes validate the proposed method, which seems very adequate to optimize future GGW structures.
\end{abstract}

Index Terms-gap technology, characteristic impedance, transmission lines, groove gap waveguide, analysis, numerical methods, rectangular waveguide, evanescent propagation.

\section{INTRODUCTION}

The millimeter-wave band is called to be the niche of a wide variety of new generation applications [1]. However, the increment of frequency is not only a matter of scale in the related microwave components. Problems such response degradation due to bad metal contacts (which is difficult to ensure in the grade of perfection for a small, millimetric wavelength), or the increase of dielectric losses, imply that component design can become a challenging task. Among other new structures, Gap Waveguides (GW) [2], based on a periodic structure and the Electromagnetic Bandgap (EBG) effect, seem to be a promising solution for these problems.

Specifically, the Groove Gap Waveguide (GGW), Fig. 1, can be an alternative to the classical rectangular waveguide (RWG) when metal contact at junctions becomes critical, since both waveguides act similarly, but the former is composed of two independent pieces that no require metal contact due to the EBG effect. Several recent designs such as Q-band slot arrays [3], V-band narrow-band filters [4], or W-band resonator and couplers [5], show promising results.

This work is supported by the Spanish Ministerio de Economia y Competitividad under projects TEC2013-47360-C3-3-P and TEC2013-47037-C5-1-R

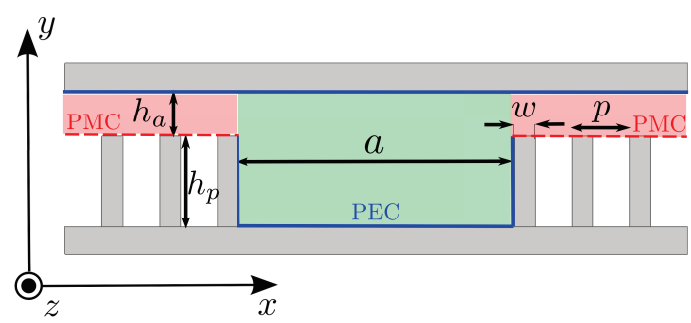

Fig. 1. GGW cross-section.

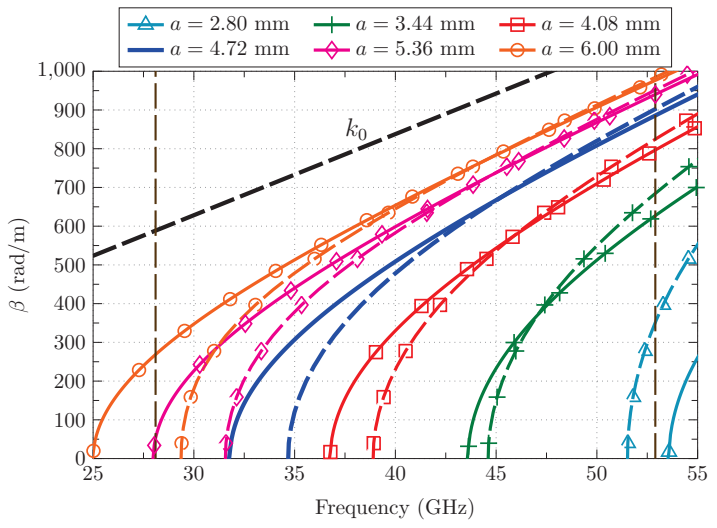

Fig. 2. Propagation constant of the RWG (solid lines) and of the GGW (dashed lines) for different values of $a$.

Unfortunately, the GGW has a complex geometry, which leads to a complicated analysis. Since the direct equivalence between RWG and a GGW with same propagation channel dimensions, [6], results in a rough approximation, optimization process requires from additional full-wave analysis, becoming the design with GGW topology a cumbersome task. Previously, some analysis methods that homogenize the periodic structure have been proposed [7]-[8]. However, on the one hand, these methods are not applicable when the periodic structure has a period comparable with the wavelength, which is usually the case for GW designs [3]-[5]. On the other hand, the information about the influence of the periodic structure geometrical parameters in the GGW behavior is lost.

In this work, a fast analysis method based on transmission line theory is presented to solve the aforementioned issue. 
The method establishes an equivalence between the GGW and the RWG which is frequency dependent, taking into account all the GGW geometrical parameters. In this way, the design possibilities are extended and good physical insight is provided due to the conceptual connection with a well-known structure such as the RWG. Furthermore, as a part of the presented work, evanescent operation of the GGW is studied for the first time to the authors' knowledge. This opens the door to the design of below-cutoff components with the GGW, which are very suitable when compactness and spurious free response are demanded [9]. The proposed approach is validated through numerical tests considering a variety of geometrical cases and two manufactured prototypes.

The paper is organized as follows, in section II, GGW principles are briefly described, and main differences between RGW and GGW are commented for the cases of above and below cutoff operation. In section III, the analysis method is presented, and several results are given. Then, in section IV, experimental validation of the proposed method is presented through two prototypes. Finally, some conclusions and remarks are given.

\section{DifFERENCES BETWEEN RWG AND GGW}

The GGW operation is based on the propagation stopband created by placing a PEC wall a distance less than $\lambda / 4$ from a PMC wall. The PMC is artificially created over a periodic structure of square metal pins (see Fig. 1). If a zone in the periodic structure is left without pins, a propagation channel (green area in Fig. 1) is created, thus having a waveguide formed by two independent pieces which indeed do not require to be in contact. The fundamental mode of this waveguide is a quasi-TE mode very similar to the $\mathrm{TE}_{10}$ of the RWG. In practice, it has been observed that the periodic structure can be truncated after the third row at each side of the structure [10].

\section{A. Operation above cutoff}

In this study, the geometrical parameters of [10] are used: $h_{p}=2.4 \mathrm{~mm} w=0.3 \mathrm{~mm}, p=0.9 \mathrm{~mm}$ and $h_{a}=0.375 \mathrm{~mm}$. These values give a stopband going from $28.1 \mathrm{GHz}$ to 52.9 $\mathrm{GHz}$, covering completely the Q-band. The simulations are carried out with $\mathrm{CST}^{\circledR}[11]$. At each side, three rows of pins are used, and a PEC boundary condition is placed where the fourth pin row should start. No influence from leaving it open has been appreciated, since the field is significantly attenuated after the third row, and this topology is more efficient computationally.

When looking at the dispersion diagrams displayed in previous works such as [4], [6] or [10], one observes that the propagation constants of the RWG and the GGW are coincident or very similar only on a narrow frequencies range, and both waveguides have a noticeable difference in the cutoff frequency. In order to better characterize the propagation properties of the GGW and the differences with regard to the RGW, a parametrization of the channel width $a$ is carried out. With six equally distributed values, going from $a=2.8 \mathrm{~mm}$

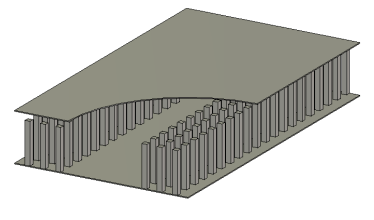

(a) Structure.

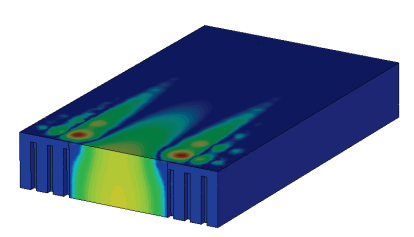

(c) $f=29 \mathrm{GHz}$.

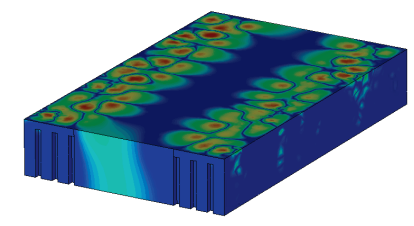

(b) $f=28 \mathrm{GHz}$.

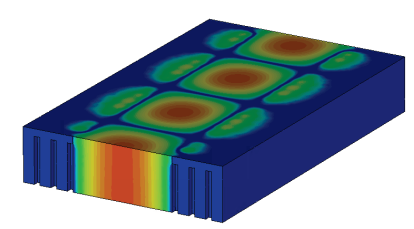

(d) $f=40 \mathrm{GHz}$.
Fig. 3. $E_{y}$ field inside a GGW with $a=4.72 \mathrm{~mm}$ for different cases of propagation.

to $a=6 \mathrm{~mm}$, the cutoff frequencies of the GGW sweep along the stopband.

The results of the parametric study are shown in Fig. 2. The standard/reference case corresponds to the value $a=4.72 \mathrm{~mm}$ [10], in blue without marks. It can be seen that the GGW is always more dispersive than the RWG, and for most of the cases, the GGW presents a higher cutoff frequency, which implies that is smaller in equivalent aperture. This could be striking, since in the GGW the fields spread beyond the propagation channel, evanescently, to the pin region. However, this cutoff difference between waveguides varies with frequency. While for large $a$ GGW has an appreciable higher cutoff than $\mathrm{RGW}$, this difference is reduced as $a$ is smaller, and for the case $a=2.8 \mathrm{~mm}$, the GGW has lower cutoff than the RWG.

This study reveals that the usual assumption of equivalence between RWG and GGW is only true under certain cases (far from cutoff and large $a$ ). Furthermore, a simple scaling factor between both waveguides is not enough, as occur between the Substrate Integrate Waveguide (SIW) and the RWG [12]. A frequency dependent behaviour is appreciated, which will be explained through the analysis of section III.

\section{B. Operation below cutoff}

In the rectangular waveguide, when observing the propation channel, the lateral conditions are PEC. Thus, below cutoff, the mode has no other option than be delivered evanescently along the axial direction. However, some doubts arise when the GGW below cutoff operation is considered. For the GGW, the lateral conditions are the forbidden propagation between pins and top plate, which also permits an exponential decay in the transversal direction [7]. It is necessary, hence, to check if the GGW is able to deliver energy evanescently in the axial direction in a similar way to the RWG.

For this analysis, it is considered $a=4.72 \mathrm{~mm}$, which implies a cutoff of $f_{c}=34.68 \mathrm{GHz}$. Since the stopband of the GGW structure starts at $f=28.1 \mathrm{GHz}$, three frequencies are analyzed, $f=28 \mathrm{GHz}$ (outside the stopband, mode below cutoff), $f=29 \mathrm{GHz}$ (inside the stopband, mode below 


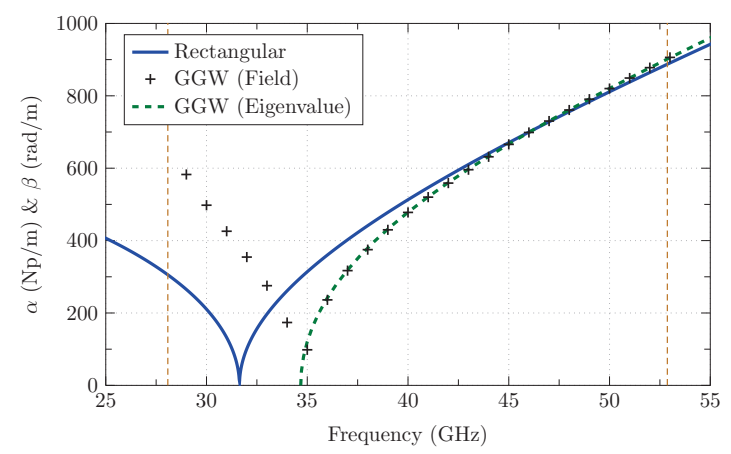

Fig. 4. Propagation and attenuation constant for a rectangular waveguide and a GGW of $a=4.72 \mathrm{~mm}$.

cutoff), and $f=40 \mathrm{GHz}$ (inside the stopband, mode under usual operation above cutoff). The results of this comparison are shown in Fig. 3. As can be seen, outside the stopband, the field spreads into the pin structure, whereas inside the stopband energy is delivered along the axial direction in a similar manner to the RWG in both cases, below and above cutoff. It is observed, however, that the field spreads more in the lateral directions when the mode is below cutoff.

In general, it is observed a larger spreading of the power to the pin regions for lower frequencies. This is explained as follows. Below cutoff, the lower the frequency, the higher the axial attenuation for the mode, which "sees" more suitable the transversal direction than for higher frequencies. Above cutoff, considering the usual decomposition of the fundamental $\mathrm{TE}_{10}$ mode in two plane waves, the higher the frequency, the closer $\beta$ to $k_{0}$, and the lower the angle of incidence.

At this point, the dispersion diagram of the GGW under below cutoff operation is studied. Whereas above cutoff an eigenvalue analysis is admitted by the commercial solver to calculate the propagation constant, for below cutoff operation (attenuation constant) the whole structure must be simulated. Once solved the fields inside the structure, the following expression is evaluated:

$$
\alpha(\mathrm{Np} / \mathrm{m})=\frac{\ln \left(\frac{E_{y}\left(z_{1}\right)}{E_{y}\left(z_{2}\right)}\right)}{z_{1}-z_{2}}
$$

where $z_{1}>z_{2}$ and $E_{y}\left(z_{i}\right)$ is the amplitude of the $E_{y}$ field component in the center of the waveguide $(x=a / 2)$ at the corresponding z-position. Of course, by analyzing the phase, $\beta$ can be also recovered for frequencies above cutoff.

The results of this dispersion analysis are shown in Fig. 4, where it is appreciated that the difference between the RWG and the GGW continue increasing when the operating frequency goes below cutoff. For this situation, the equivalence between waveguides results in a quite poor approximation.

\section{ANALYSIS METHOD}

\section{A. Proposed Model}

The proposed model starts analyzing the GGW in the transversal direction, i.e, assuming propagation along $\hat{x}$, see

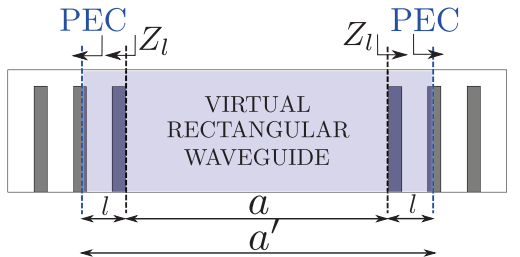

(a) Proposed method

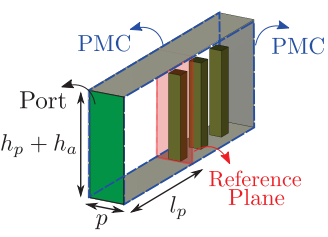

(b) $\mathrm{CST}^{\circledR}$ model.
Fig. 5. Conceptual and simulation schematics of the proposed method.

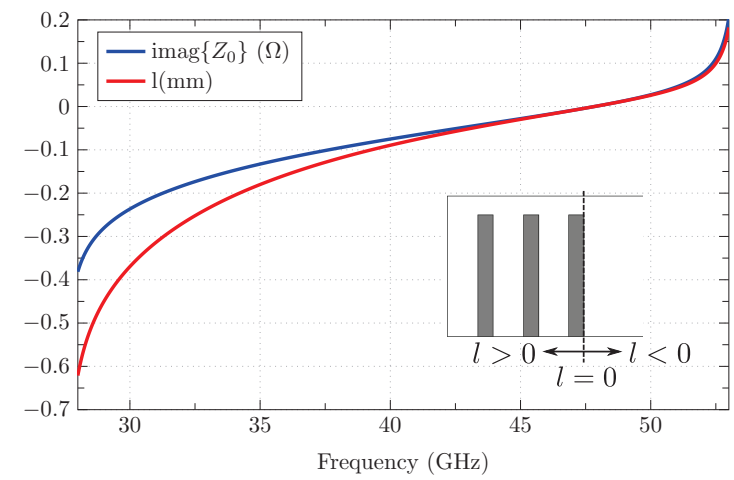

Fig. 6. Lateral impedance viewed at the first pin row plane, and the distance at which a PEC wall would produce the same impedance.

Fig. 5. In this configuration, it makes sense to consider the impedance of the waves incident on the side wall, $Z_{l}$. Due to symmetry and periodicity, respectively, only one side and one period should be taken into account. The simulated structure for this analysis is shown in Fig. 5(b). Periodicity is achieved by means of PMC planes and image theory. The structure is terminated with a PEC, located at a distance $s=p-w$ from the third pin. No differences are appreciated by leaving it open or considering more than three pin rows.

If the structure of Fig. 5(b) is solved, and the $S_{11}$ parameter is de-embedded to the reference plane, one obtains the normalized input impedance at this plane as

$$
Z_{l n}=\frac{1+S_{11}}{1-S_{11}}
$$

By means of transmission line theory, the same impedance could be observed at this reference plane if the pins are replaced by a shorted stub, with the short placed at an appropriate distance

$$
l=-\frac{1}{\beta_{p}} \tan ^{-1}\left(j Z_{l n}\right)
$$

where $\beta_{p}$ is the propagation constant of the lateral parallel plate waveguide feeding the structure with three pins (see the port in green in Fig. 5(a) and consider periodicity). Going back to Fig. 5(a), one realizes that this equivalence with a shorted stub corresponds to having a virtual rectangular waveguide of width $a^{\prime}=a+2 l$. This is indeed the proper equivalent for the GGW. 


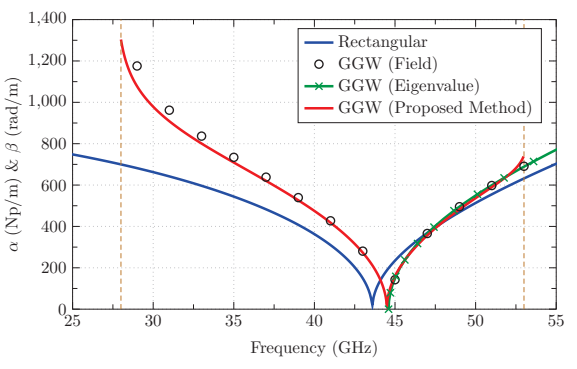

(a) $a=3.44 \mathrm{~mm}$.

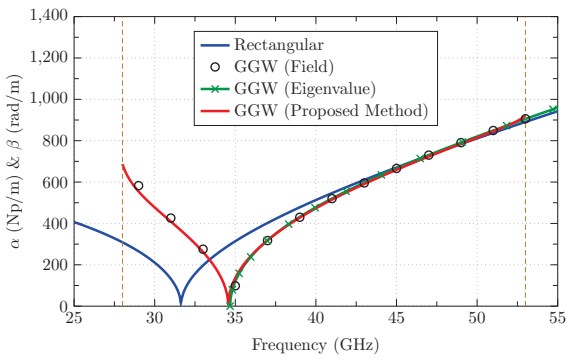

(b) $a=4.72 \mathrm{~mm}$.

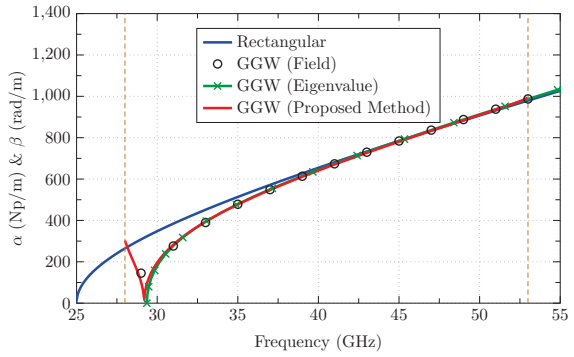

(c) $a=6.00 \mathrm{~mm}$.

Fig. 7. Propagation and attenuation constants of the rectangular waveguide and the GGW, comparing several calculations methods for the GGW case.

Fig. 6 shows the imaginary part of the impedance $Z_{l}$ (note that $Z_{l}$ is a reactance since a lossless structure is considered) and the corresponding $l$ through (3). The equivalent obtained is a RWG whose width grows with the frequency.

\section{B. Results}

To test the proposed method, the cases from Fig. 2 have been evaluated, showing good agreement. Results for some of these cases are shown in Fig. 7. As can be observed, the frequency dependent equivalent RWG used in the proposed method is able to recover with good accuracy the dispersion curves of the GGW in both, below and above cutoff regions. Furthermore, this approach gives an interesting physical insight that explains the GGW behavior. Consider the propagation and attenuation constants formulas for the RWG:

$$
\begin{array}{rlrl}
\beta & =\sqrt{k^{2}-\left(\frac{\pi}{a^{\prime}}\right)^{2}} & & f \geq f_{c} \\
\alpha & =\sqrt{\left(\frac{\pi}{a^{\prime}}\right)^{2}-k^{2}} \quad & f<f_{c}
\end{array}
$$

Above cutoff, as $a^{\prime}=a+2 l(f)$ grows with frequency (4) implies for GGW that $\beta$ grows with the frequency faster than for the rectangular waveguide case. When the term $k^{2}$ is large compared with $\left(\pi / a^{\prime}\right)^{2}$ the variation of $a^{\prime}$ is less significant, and the propagation behavior is similar to that of a standard rectangular waveguide. This occurs for large values of $a$ and high frequencies (as previously observed in Fig. 2).

Below cutoff, as frequency is reduced, the term $k^{2}$ becomes small compared with $\left(\pi / a^{\prime}\right)^{2}$. Thus, with regard to (5), $\alpha$ exhibits growth with the decrease of $a^{\prime}$ with the frequency. This effect explains why the $\alpha$ curve of the GGW does not exhibit a reduction of its slope, as occurs with the rectangular waveguide when the frequency decreases, and the accentuated difference between both waveguides in this region.

It is worth to mention that due to Foster Reactance Theorem [13], the lateral reactance will always monotonically increase with the frequency, and hence $l$. This implies that GGW will always exhibit greater dispersion than the RWG.

Now, the robustness of the presented method is tested by considering two additional extreme cases, A and B (see Fig. 8), which correspond, respectively, to a very dispersive GGW

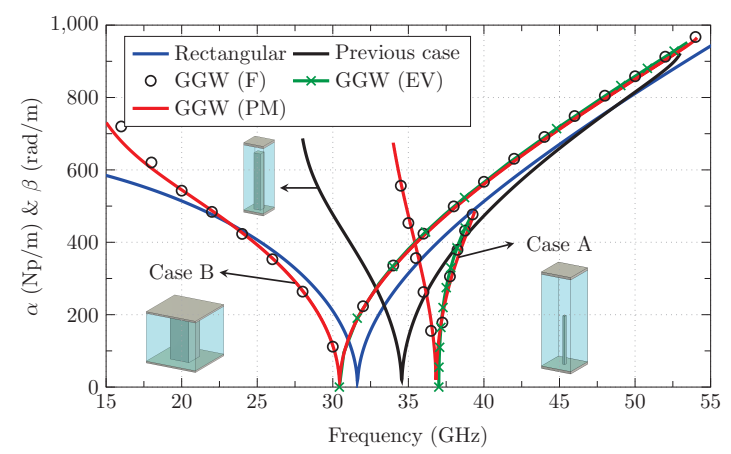

Fig. 8. Propagation and attenuation constants of a GGW with $a=4.72$ $\mathrm{mm}$ for two different cases (A and B) of the pin structure, comparing several calculation methods (field -F-, eigenvalue -EV-, proposed method -PM-). Case A: $w=0.1 \mathrm{~mm}, p=1.3 \mathrm{~mm}, h_{p}=2 \mathrm{~mm}$, and $h_{a}=1.8 \mathrm{~mm}$. Case B $w=1.15 \mathrm{~mm}, p=3 \mathrm{~mm}, h_{p}=2.6 \mathrm{~mm}$, and $h_{a}=0.1 \mathrm{~mm}$. Rectangular waveguide curves and previous GGW curves have been added for comparison. Also, images of the unit cells of the different geometries are included. GGW curves are only depicted inside the corresponding stopband.

and a GGW with lower dispersion. Results are also shown in Fig. 8. The good agreement obtained also in those extreme cases indicates that this method is relevant to a wide variety of geometries of the periodic structure. Furthermore, it is observed that the GGW offer interesting dispersion features. Since the structure of Fig. 5(b) is quickly solved, as it will be shown next, the presented method could be used for dispersion engineering purposes by optimization of the periodic structure geometry.

To end this section, a computation efficiency evaluation is carried out for the original case with $a=4.72 \mathrm{~mm}$. The used computing machine incorporates an Intel ${ }^{\circledR}$ Xenon ${ }^{\circledR}$ CPU E31245 @ $3.40 \mathrm{GHz}$ and 16 GB of RAM memory. Results shown in the Table I correspond to the computation times given by $\mathrm{CST}^{\circledR}$ for each case.

The presented method is not only faster than the other calculations, but same results can be used for different $a$ values, since $Z_{l}$ only depends on the pin structure. This feature results very interesting for component optimization.

\section{EXPERIMENTAL VALIDATION}

Experimental validation of the presented results has been done by means of two TRL calibration kits corresponding 
TABLE I

COMPARISON OF CPU TIMES.

\begin{tabular}{cccc}
\cline { 2 - 4 } Computation time & Field & Eigenvalue & Proposed Method \\
\cline { 2 - 4 } & $1380 \mathrm{~s}$ & $4230 \mathrm{~s}$ & $24 \mathrm{~s}$
\end{tabular}

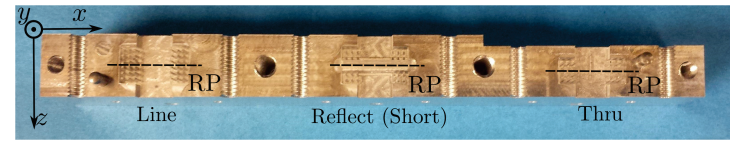

Fig. 9. TRL calibration kit. Bottom piece containing the pins.

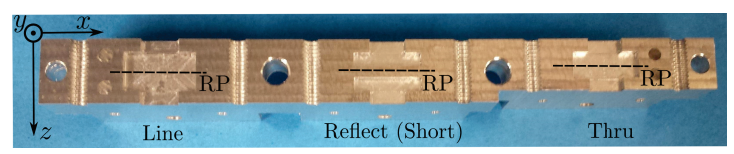

Fig. 10. TRL calibration kit. Top piece.

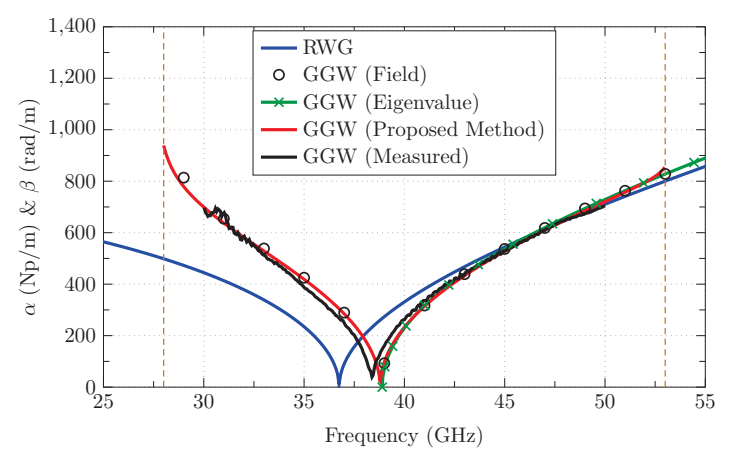

Fig. 11. Comparison between simulated and measured results, $a=4.08 \mathrm{~mm}$.

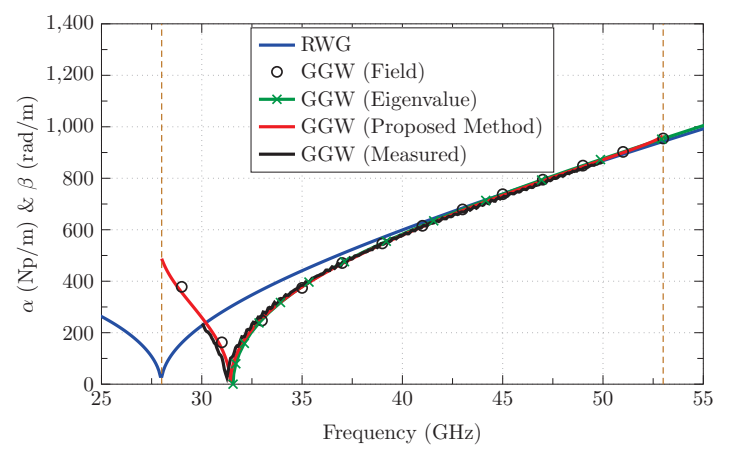

Fig. 12. Comparison between simulated and measured results, $a=5.36 \mathrm{~mm}$

to the GGW widths $a=4.08 \mathrm{~mm}$ and $a=5.36 \mathrm{~mm}$, manufactured through an in-house process using a DATRON ${ }^{\circledR}$ M25 milling system. Fig. 9 and 10 show the top and bottom pieces, respectively, for one of the fabricated calibration kits. Since the TRL calibration algorithm allows to recover the complex propagation constant $\gamma=\alpha+j \beta$ of each waveguide, no additional components are required.

Simulated and experimental results are compared in Figs. 11 and 12. It is observed good agreement between curves, with only slight frequency shift, justified by the limited precision of the in-house process, which is estimated to be about $\pm 10 \mu \mathrm{m}$.

\section{CONCLUSiON}

This paper has shown that the direct equivalent correspondence normally assumed between the GGW and rectangular waveguide, which is commonly used in practice, is a rough approximation providing accurate results only for some specific cases. Also, for the first time, the behaviour of GGW below cutoff has been studied. This study has shown that the GGW and the rectangular waveguide behave in a very different way in terms of their dispersion characteristics. A simple method for the accurate analysis of GGW dispersion characteristics, based on equivalent short-circuited transmission lines, has been presented, and it has been shown to have very good prediction capability for all frequencies (below and above cutoff), and different pin geometries. The proposed model has a significantly reduced computational effort, thus being suitable for fast parametric analysis of GGWs and their efficient design through optimization algorithms. Using the proposed model it is deduced that the GGW is equivalent to a virtual rectangular waveguide whose width grows with the frequency. Two TRL calibration kits have been manufactured to obtain experimentally the dispersion curves of two GGWs. The good agreement observed between the measured and simulated results fully validates the proposed method.

\section{REFERENCES}

[1] D. Lockie and D. Peck, "High-data-rate millimeter-wave radios," IEEE Microwave Magazine, vol. 10, no. 5, pp. 75-83, 2009.

[2] P.-S. Kildal, "Three metamaterial-based gap waveguides between parallel metal plates for mm/submm waves," in 3rd European Conference on Antennas and Propagation (EuCAP), 2009, pp. 28-32.

[3] J. Herranz-Herruzo, A. Valero-Nogueira, S. Martinez-Giner, and A. Vila Jimenez, "Untilted narrow-wall slots excited by parasitic dipoles in groove-gap waveguide technology," IEEE Transactions on Antennas and Propagation, vol. PP, no. 99, pp. 1-1, 2015.

[4] A. Berenguer, M. Baquero-Escudero, D. Sanchez-Escuderos, B. Bernardo-Clemente, and V. E. Boria-Esbert, "Low insertion loss 61 ghz narrow-band filter implemented with groove gap waveguides," in 45th European Microwave Conference (EuMC). IEEE, 2014, pp. 1-4.

[5] S. Rahiminejad, H. Raza, A. Zaman, S. Haasl, P. Enoksson, and P.-S. Kildal, "Micromachined gap waveguides for $100 \mathrm{GHz}$ applications," in 7th European Conference on Antennas and Propagation (EuCAP), 2013, pp. 1935-1938.

[6] H. Raza, J. Yang, P.-S. Kildal, and E. Alfonso, "Resemblance between gap waveguides and hollow waveguides," IET Microwaves, Antennas Propagation, pp. 1-7, 2013.

[7] A. Polemi and S. Maci, "Closed form expressions for the modal dispersion equations and for the characteristic impedance of a metamaterialbased gap waveguide," IET Microwaves, Antennas \& Propagation, vol. 4, no. 8, pp. 1073-1080, 2010.

[8] M. Bosiljevac, A. Polemi, S. Maci, and Z. Sipus, "Analytic approach to the analysis of ridge and groove gap waveguides-comparison of two methods," in 5th European Conference on Antennas and Propagation (EUCAP). IEEE, 2011, pp. 1886-1889.

[9] G. F. Craven and R. F. Skedd, Evanescent mode microwave components, 1987, vol. 1.

[10] A. del Olmo-Olmeda, M. Baquero-Escudero, V. E. Boria-Esbert, A. Valero-Nogueira, and A. Berenguer, "A novel band-pass filter topology for millimeter-wave applications based on the groove gap waveguide," in International Microwave Symposium, 2013, pp. 1-4.

[11] C. C. S. Technology, "CST studio suite," 2015, Suite 2015, Germany.

[12] W. Che, K. Deng, D. Wang, and Y. Chow, "Analytical equivalence between substrate-integrated waveguide and rectangular waveguide," IET microwaves, antennas \& propagation, vol. 2, no. 1, pp. 35-41, 2008

[13] R. M. Foster, "A reactance theorem," Bell System Technical Journal, vol. 3, no. 2, pp. 259-267, 1924. 